\title{
Suco de laranja reduz o colesterol em indivíduos normolipidêmicos ${ }^{1}$
}

\author{
Cholesterol-lowering effect of orange \\ juice in normolipidemic subjects
}

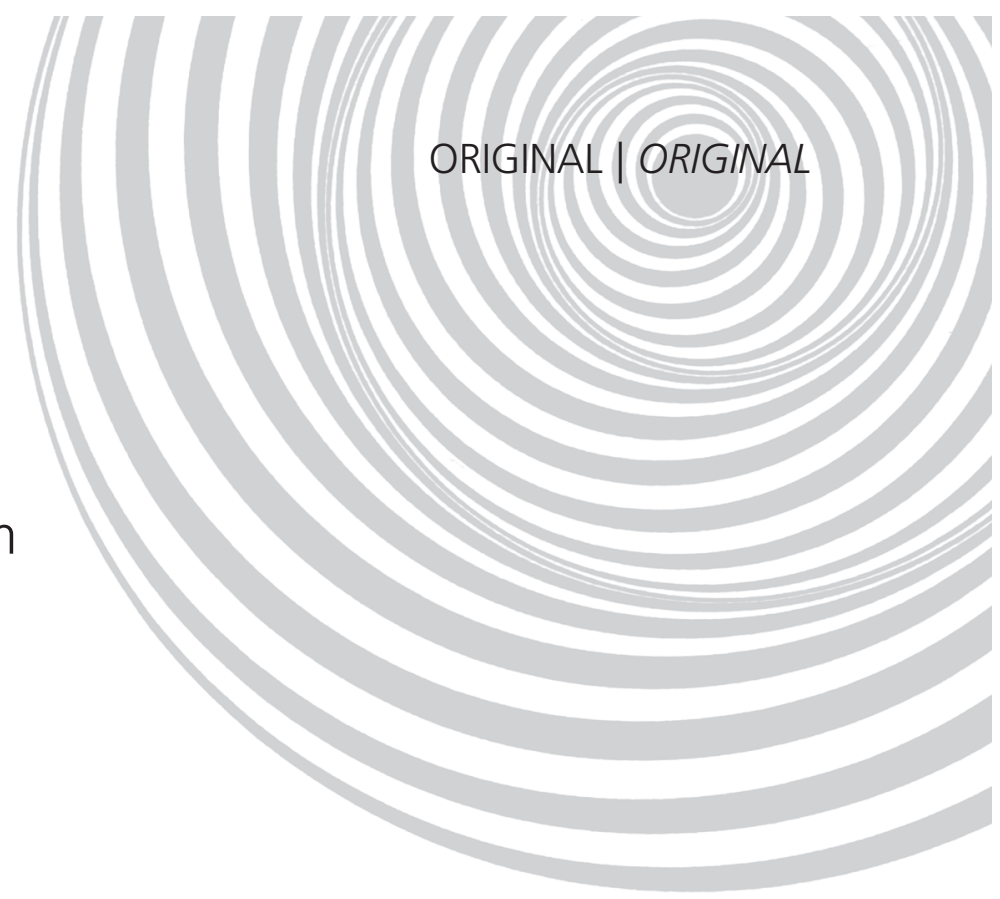

Thais Borges CÉSAR2

Layane Urzedo RODRIGUES 3

Milena Salomão Peres de ARAÚJO3

Nancy Preising APTEKMANN ${ }^{3}$

RE S U M O

\section{Objetivos}

Neste estudo foi investigado o efeito do consumo habitual de suco de laranja no perfil dos lípides e lipoproteínas em homens e mulheres normolipidêmicos.

\section{Métodos}

Todos os voluntários $(n=29)$ consumiram $750 \mathrm{~mL} /$ dia de suco de laranja durante 60 dias. Variáveis bioquímicas como perfil lipídico, apolipoproteínas, glicose, paraoxonase1 e o tamanho de HDL foram medidas antes e após o período de suplementação com suco de laranja. Também foram realizadas medidas antropométricas e inquéritos dietéticos.

\section{Resultados}

O consumo crônico de suco de laranja reduziu significativamente o colesterol total nos homens $(11 \%, p<0,05)$ e nas mulheres $(10 \%, p<0,05)$ e o LDL-C nos homens e mulheres $(15 \%, p<0,05)$. O HDL-C e a apoA-I também diminuíram, refletindo a redução do colesterol total. Os triacilgliceróis, apo B, PON1, tamanho da HDL, IMC, gordura corporal e circunferência abdominal não foram modificados com o tratamento com suco de laranja.

\section{Conclusão}

Neste estudo, mostrou-se que o suco de laranja apresenta propriedade redutora sobre o colesterol, e foi sugerido que a associação dos flavonóides cítricos com a vitamina C previne o estresse oxidativo e o desenvolvimento da aterosclerose.

Termos de indexação: Colesterol. Colesterol-LDL. Lipoproteínas. Suco.

1 Artigo elaborado a partir da dissertação de L.U. RODRIGUES, intitulada "Suco de laranja reduz colesterol e insulina no sangue de indivíduos saudáveis". Universidade Estadual Paulista Júlio de Mesquita Filho; 2008

2 Universidade Estadual Paulista Júlio de Mesquita Filho, Faculdade de Ciências Farmacêuticas, Departamento de Alimentos e Nutrição. Rod. Araraquara-Jaú, Km 1, 14801-902, Araraquara, SP, Brasil. Correspondência para/Correspondence to: T.B. CÉSAR. E-mail: <tcesar@fcfar.unesp.br>

${ }^{3}$ Universidade Estadual Paulista Júlio de Mesquita Filho, Faculdade de Ciências Farmacêuticas. Araraquara, SP, Brasil. 


\section{A B S T R A C T}

\section{Objective}

This study investigated the effect of regular orange juice consumption on blood lipids and lipoproteins of normolipidemic men and women.

\section{Methods}

All volunteers ( $n=29$ ) consumed $750 \mathrm{~mL}$ of orange juice daily for 60 days. Biochemical variables such as lipid profile, apolipoproteins, glucose, paraoxonase 1, and HDL size, were measured before and after the orange juice consumption period. Anthropometric and dietary data were also collected.

\section{Results}

Daily consumption of orange juice significantly reduced total cholesterol in men $(11 \%, p<0.05)$ and women $(10 \%, p<0.05)$ and LDL-cholesterol $(15 \%, p<0.05)$ in men and women. HDL-C and Apo A-I also decreased, reflecting the reduction in total cholesterol. Triacylglycerols, apolipoprotein B, PON 1, HDL size, BMI, body fat, and waist circumference were not affected by orange juice consumption.

\section{Conclusion}

Orange juice presented cholesterol-lowering activity and the association between citrus flavonoids and vitamin C may prevent oxidative stress and the development of atherosclerosis.

Indexing terms: Cholesterol. Cholesterol-LDL. Lipoproteins. Juice.

\section{N T R O D U Ç Ã O}

Estudos atuais têm mostrado que os flavonóides, um grupo de substâncias não nutritivas presentes nos alimentos, podem retardar ou prevenir as doenças ateroscleróticas ${ }^{1}$ devido à atividade antioxidante sobre as Lipoproteínas de Baixa Densidade (LDL) e consequente ação inibidora na formação da placa ateromatosa². Os flavonóides são compostos naturais encontrados em frutas e vegetais e por isso extensivamente consumido por vegetarianos e grupos de indivíduos com amplo consumo de frutas e vegetais, levando à menor incidência da doença aterosclerótica nessas populações ${ }^{3}$. O estudo da atividade metabólica dos flavonóides tem demonstrado ainda a atenuação do estresse oxidativo em doenças como o câncer, diabetes mellitus, doenças neurodegenerativas, aterosclerose, entre outras ${ }^{1,4,5}$.

O suco de laranja é um alimento singular devido ao alto conteúdo de vitamina $\mathrm{C}$ e a quantidades apreciáveis de outros nutrientes essenciais, como carotenóides, folato e potássio. As frutas cítricas, incluindo o suco de laranja, são fontes dos flavonóides hesperidina e naringinina, que protegem contra o câncer ${ }^{6}$ e a aterosclerose ${ }^{5}$.
Outro estudo relatou a associação dos flavonóides cítricos ou flavanonas com redução do risco de Doença Arterial Coronariana (DAC), redução do colesterol sanguíneo, inibição da oxidação da LDL e ácidos graxos e ainda redução da agregação plaquetária ${ }^{7}$. A hesperidina é o principal componente flavonóide encontrado exclusivamente nas frutas cítricas e em quantidades apreciáveis no suco de laranja ${ }^{8}$. A hesperidina juntamente com a naringenina, o segundo mais importante flavonóide das frutas cítricas, exibem ação cardioprotetora indireta por exercer efeito supressor sobre as espécies reativas de oxigênio in vitro ${ }^{9}$.

No homem e em animais experimentais, o suco de laranja e a hesperidina isolada mostraram efeito hipolipidêmico sobre o colesterol total, LDL-Colesterol (LDL-C) e triacilgliceróis, e aumento do colesterol da Lipoproteína de Alta Densidade $(\mathrm{HDL}-\mathrm{C})^{10,11}$. Foi sugerido que as flavanonas são capazes de reduzir os níveis de colesterol sanguíneo por dois mecanismos básicos: 1) inibição da Enzima Acetil-Coenzima-A Acil Transferase (ACAT), responsável pela esterificação do colesterol hepático, e 2) aumento da atividade dos receptores celulares de LDL-C ${ }^{12}$.

Estudos epidemiológicos têm que a vitamina C contida nas frutas e nos vegetais se associa 
com a redução do risco de $\mathrm{DAC}^{13}$, embora resultados conflitantes tenham sido observados em estudos com suplementação de vitamina C e mortalidade por DAC ${ }^{14}$. Porém, a terapêutica com doses elevadas de vitamina $C$ melhorou a função vascular de pacientes com doença arterial coronariana $^{15}$, e a ingestão crônica com o suplemento melhoraram a função endotelial ${ }^{16}$. Outro estudo atribuiu à vitamina $C$ do suco de laranja ação antioxidante em mulheres que consumiram $600 \mathrm{~mL}$ de suco por dia ${ }^{17}$. Foi ainda observado que $500 \mathrm{~mL}$ de suco de laranja diariamente aumentou a concentração de vitamina $C$ e reduziu os radicais livres no sangue de homens ${ }^{18,19}$.

A hipótese oxidativa da aterosclerose tem contribuído para ampliar o campo de estudo das substâncias antioxidantes que podem prevenir o estresse oxidativo, reduzir a oxidação da LDL e inibir o desenvolvimento da aterosclerose ${ }^{20}$. Um agente antioxidante natural do organismo humano é a enzima Paraoxonase 1 (PON1), que possui papel fundamental na prevenção da aterosclerose ao impedir a oxidação da LDL-C e HDL-C e inativação de lípides oxidados da $L D L^{21}$. Identificada em tecidos animais em 1946 por Abraham Mazur, a PON1 é uma esterase/lactonase associada à $\mathrm{HDL}^{22}$, e sua atividade está inversamente relacionada ao risco de doenças cardiovasculares ${ }^{21}$. Experimentos in vitro mostraram que a PON1 pode proteger a HDL da oxidação, preservando a integridade da partícula, e atenuar a oxidação da LDL, por hidrolisar os lipídeos oxidados nas lipoproteínas, nos macrófagos e nas lesões ateroscleróticas $^{23}$. Mas para exercer seu efeito antioxidante é necessário que haja interação entre a PON1 e os lipídeos oxidados, o que pode ocorrer de duas formas: (1) por difusão da PON1 durante a interação entre LDL e HDL; e (2) provável transferência dos lipídeos oxidados da LDL para a HDL e posterior ação da PON122.

Este estudo se propôs a investigar o efeito crônico da ingestão de suco de laranja sobre alguns fatores de risco para o desenvolvimento das doenças cardiovasculares. Para tanto, foram avaliados o perfil dos lípides sanguíneos, apolipo- proteínas, PON 1, tamanho da partícula de HDL, variáveis antropométricas e ingestão alimentar de homens e mulheres adultos submetidos à suplementação com suco de laranja.

\section{M É T O D O S}

As medidas antropométricas, dietéticas e bioquímicas foram realizadas em um grupo de 34 indivíduos saudáveis, recrutados na Faculdade de Ciências Farmacêuticas da Universidade Estadual Paulista Júlio de Mesquita Filho (Unesp) e na comunidade de Araraquara (SP). O grupo foi composto por 17 homens e 17 mulheres, com idade entre 25 e 55 anos; destes, apenas 3 homens e 2 mulheres não finalizaram o estudo. Os voluntários foram informados acerca da finalidade do trabalho e as dúvidas foram esclarecidas. Foi solicitado aos voluntários que não modificassem seus hábitos de alimentação ou de atividade física, quando houvesse, durante todo o período experimental. A seleção foi realizada de acordo com as normas do Comitê de Ética em Pesquisa da Faculdade de Ciências Farmacêuticas da Unesp de Araraquara (SP), protocolo CEP/FCFCAr n 8/2005. Após a aceitação do termo de consentimento livre e esclarecido, procedeu-se ao início do experimento.

Avaliação antropométrica: Os participantes foram pesados e medidos em dois momentos do estudo: antes e após o tratamento com suco de laranja durante 60 dias. A massa corporal foi obtida com o indivíduo imóvel, sem sapatos e com roupas leves, em balança mecânica modelo 31 Filizola ${ }^{\circledR}$, com capacidade de $150 \mathrm{~kg}$ e divisão de 100g. A estatura foi registrada em metros e obtida a partir da fixação em superfície vertical de fita métrica não extensível, a $100 \mathrm{~cm}$ do chão; os participantes em pé, descalços, com os calcanhares juntos, costas retas e braços estendidos ao lado do corpo para a obtenção da medida. A classificação do estado nutricional foi realizada segundo o Índice de Massa Corporal (IMC) ${ }^{24}$. A medida da circunferência da cintura, tomada na cicatriz umbilical, foi realizada com uma fita métrica não 
extensível, com o indivíduo em pé. A medida foi feita no momento da expiração e os resultados foram interpretados com base nos valores limítrofes de $94 \mathrm{~cm}$ para os homens e $80 \mathrm{~cm}$ para as mulheres ${ }^{24}$. O percentual de gordura corpórea foi obtido por bioimpedância elétrica com o equipamento "Biodinamics, Modelo 310", da Biodinamics Corp, EUA, aplicado pela manhã com os participantes em jejum, sem atividade física e após repouso de 15 minutos ${ }^{25}$.

Avaliação dietética: Constou da aplicação de dois recordatórios de 24 horas sendo um em dia da semana e outro em um dia do final de semana, aplicados nos períodos anterior e posterior à suplementação com suco de laranja, resultando em quatro recordatórios ao final do experimento. Os resultados foram expressos como a média do recordatórios do dia da semana e do final da semana, nos períodos pré e pós-tratamento. Os dados de energia e nutrientes dos recordatórios foram obtidos com o "Programa de Apoio à Nutrição - Nutwin", versão 1.5.2.50, 2005, Escola Paulista de Medicina, Unifesp (SP), Brasil. A adequação nutricional dos macronutrientes foi avaliada de acordo com as Recomendações Nutricionais ${ }^{26}$. O consumo diário de $750 \mathrm{~mL}$ de suco de laranja foi avaliado semanalmente por ocasião da entrega do suco concentrado aos participantes, tendo sido verificada uma adesão superior a $85,0 \%$ em todos os casos analisados. Durante o período experimental, cinco indivíduos (três homens e duas mulheres) que não conseguiram tomar a dose diária pré-determinada de $750 \mathrm{~mL} /$ dia foram encorajados a abandonar o estudo. A atividade física dos participantes foi obtida pela descrição individual dos hábitos e frequência diária de exercícios, caminhada, atividades de lazer, entre outros, antes e após o período experimental e foi avaliada de acordo com os níveis de atividade física utilizados no cálculo do gasto energético ${ }^{26}$.

Suco de Laranja: Os participantes ingeriram $750 \mathrm{~mL}$ de suco de laranja diariamente durante 60 dias ininterruptos. A dose oferecida foi baseada em estudo realizado com doses crescen- tes de suco de laranja (250, 500 e $750 \mathrm{~mL} /$ dia) oferecidas a homens, que mostrou que somente a maior dose $(750 \mathrm{~mL} /$ dia) provocou modificações significativas no perfil lipídico ${ }^{11}$, e por isso foi escoIhida para aplicação no presente experimento. 0 suco concentrado congelado foi cedido pela empresa Citrosuco Paulista S.A., pertencente ao Grupo Fischer (Matão-SP). Um único lote de suco de laranja concentrado e congelado (64,89 Brix e Ratio 17,12), obtido por processo industrial de extração do suco e concentrado por centrifugação, foi retirado na fábrica em baldes de $26 \mathrm{~kg}$. 0 suco concentrado foi envasado assepticamente em garrafas de $900 \mathrm{~mL}$ que foram armazenadas a $-20^{\circ} \mathrm{C}$. Os participantes foram instruídos a retirar semanalmente no laboratório experimental uma garrafa do suco concentrado $(900 \mathrm{~mL})$, e a diluir diariamente $125 \mathrm{~mL}$ com $625 \mathrm{~mL}$ de água filtrada em recipiente plástico graduado, fornecido previamente, que deveria ser mantido tampado em geladeira até o completo consumo. Foi recomendado o consumo de suco sem açúcar, distribuído ao longo do dia durante as principais refeições. O suco diluído, sem adição de açúcar, continha um teor de sólidos solúveis de $12^{\circ}$ Brix, 19\% de polpa e $\mathrm{pH} 3,73$. Os principais nutrientes em $750 \mathrm{~mL}$ de suco de laranja, pronto para beber, de acordo com as especificações técnicas do produto, foram: $315 \mathrm{kcal}, 42 \mathrm{mg}$ de hesperitina, $12 \mathrm{mg}$ de naringenina, $64 \mathrm{~g}$ de açúcares totais $(2: 1: 1$ de sacarose: frutose: glicose), $1308 \mathrm{mg}$ de potássio, 258mg de vitamina C e 135mg de ácido fólico.

Avaliação bioquímica: As colheitas de sangue dos participantes foram realizadas em duas ocasiões - no início do experimento e após 60 dias da suplementação com suco de laranja - no Laboratório de Análises Clínicas São Lucas, Araraquara (SP), com os indivíduos em jejum de 12 horas e em repouso. As amostras de sangue foram centrifugadas para separação do soro e em seguida realizadas as determinações bioquímicas. As amostras separadas para análises da PON 1 e tamanho da partícula de HDL foram encaminhadas ao Laboratório de Lípides do Instituto do 
Coração (InCor), São Paulo (SP). Todas as dosagens bioquímicas foram realizadas em equipamento automático, modelo System Vitros Chemistry 250, Brasil. As apolipoproteínas A-I e B (apo A-I e apo B) foram dosadas por imunoturbidimetria automatizada, com kits comerciais Roche (Apolipoproteínas Al e B, Imunotubidimétrico, Hitachi 912, Roche Diagnostics). O colesterol total e os triacilgliceróis foram determinados pelo método Enzimático-Trinder, utilizando-se o kit comercial Labtest (Colesterol Liquiform Ref. 76, Colesterol oxidase-Reação de Trinder, Triacilgliceróis Liquiform Ref. 87, Glicerol fosfato oxidase, Labtest Diagnóstica). O HDL-C foi determinado por Inibição Seletiva, sistema para determinação homogênea direta do colesterol HDL no soro humano, utilizando o kit comercial Labtest (Colesterol HDL Ref. 13. Precipitação com fosfotungstato-magnésio, Labtest Diagnóstica). O valor de LDL-C foi obtido a partir dos resultados das dosagens do colesterol total, triacilgliceróis e HDL-C, pela fórmula de Friedewald ${ }^{27}$. A glicose foi determinada pelo método enzimático GOD-Trinder, utilizando o kit comercial Glicose Pap Liquiform 1 × 500mL Labtest (Glicose HK Liquiform Ref. 85, Hexokinase de acordo com recomendação da IFCC). O tamanho da HDL foi mensurado segundo a técnica de laser de dispersão ${ }^{28}$.

Análise estatística: Os resultados foram analisados por meio do software Microsoft Office Excel 2003 e Sigma Stat, versão 2.03, 1995. As variáveis foram apresentadas como média e desvio-padrão, e todos os conjuntos de dados foram testados quanto à normalidade de suas distri- buições. A comparação entre grupos de dados com distribuição normal foi examinada com o teste $t$ de Student e para os demais com distribuição não normal foi utilizado o teste não paramétrico de Wilcoxon. O nível de significância adotado foi de $5 \%$ para as comparações efetuadas.

\section{RESULTA DOS}

No início do experimento, cerca de 36,0\% dos homens e 33,0\% das mulheres apresentavam peso corporal acima da faixa de normalidade: sobrepeso e obesidade. Foi verificada uma alta incidência de indivíduos com uma dieta rica em gorduras saturadas e colesterol, e sem hábitos de atividade física, sugerindo um estilo de vida sedentário e maior risco para as doenças cardiovasculares. Considerando esses fatores e para uma análise mais acurada dos resultados, os voluntários foram divididos em quatro grupos, de acordo com seu perfil antropométrico e gênero, a saber: (1) homens eutróficos e (2) homens com sobrepeso, (3) mulheres eutróficas e (4) mulheres com sobrepeso.

Contrariamente à suposição de que o suco de laranja leva ao aumento do peso corporal por causa do seu conteúdo energético, o elevado consumo de suco de laranja neste estudo de $750 \mathrm{~mL} /$ dia, equivalente a $315 \mathrm{kcal}$, não foi associado com nenhuma variação detectável no peso, IMC, porcentagem de gordura corporal e circunferência da cintura após a suplementação em todos os grupos analisados (Tabela 1).

Tabela 1. Variáveis antropométricas em mulheres $(n=15)$ e homens $(n=14)$ distribuídas de acordo com o índice de massa corporal (IMC) no início do experimento (pré) e ao final (pós) da suplementação com suco de laranja. Araraquara (SP), 2006.

\begin{tabular}{|c|c|c|c|c|c|c|c|c|c|c|c|c|c|c|c|c|}
\hline \multirow{4}{*}{$\begin{array}{l}\text { Peso } \\
\text { Suco laranja }\end{array}$} & \multicolumn{8}{|c|}{ Mulheres } & \multicolumn{8}{|c|}{ Homens } \\
\hline & \multicolumn{4}{|c|}{$\begin{array}{l}\text { Eutrofia }\left(\leq 25 \mathrm{~kg} / \mathrm{m}^{2}\right) \\
(\mathrm{n}=10)\end{array}$} & \multicolumn{4}{|c|}{$\begin{array}{c}\text { Sob/Obeso }\left(>25 \mathrm{~kg} / \mathrm{m}^{2}\right) \\
(\mathrm{n}=5)\end{array}$} & \multicolumn{4}{|c|}{$\begin{array}{c}\text { Eutrofia }\left(\leq 25 \mathrm{~kg} / \mathrm{m}^{2}\right) \\
\qquad(\mathrm{n}=9)\end{array}$} & \multicolumn{4}{|c|}{$\begin{array}{c}\text { Sob/Obeso }\left(>25 \mathrm{~kg} / \mathrm{m}^{2}\right) \\
(\mathrm{n}=5)\end{array}$} \\
\hline & \multicolumn{2}{|c|}{ Pré } & \multicolumn{2}{|c|}{ Pós } & \multicolumn{2}{|c|}{ Pré } & \multicolumn{2}{|c|}{ Pós } & \multicolumn{2}{|c|}{ Pré } & \multicolumn{2}{|c|}{ Pós } & \multicolumn{2}{|c|}{ Pré } & \multicolumn{2}{|c|}{ Pós } \\
\hline & $M$ & DP & $\mathrm{M}$ & DP & $\mathrm{M}$ & DP & $\mathrm{M}$ & $\overline{D P}$ & $\mathrm{M}$ & $\mathrm{DP}$ & $M$ & $\mathrm{DP}$ & $\mathrm{M}$ & $\overline{D P}$ & $M$ & DP \\
\hline Peso $(\mathrm{kg})$ & 58 & 7 & 57 & 7 & 70 & 7 & 70 & 5 & 66 & 5 & 67 & 5 & 93 & 16 & 93 & 16 \\
\hline IMC $\left(\mathrm{kg} / \mathrm{m}^{2}\right)$ & 21 & 2 & 21 & 2 & 28 & 3 & 28 & 3 & 22 & 1 & 22 & 1 & 30 & 3 & 30 & 3 \\
\hline Gordura corporal (\%) & 28 & 4 & 26 & 4 & 34 & 4 & 34 & 3 & 22 & 6 & 21 & 3 & 27 & 5 & 21 & 6 \\
\hline Circunferência abdominal (cm) & 77 & 6 & 77 & 5 & 90 & 7 & 90 & 7 & 79 & 7 & 79 & 7 & 104 & 13 & 104 & 12 \\
\hline
\end{tabular}


A comparação do consumo de alimentos ricos em lípides, ácidos graxos saturados e colesterol no início e no final do experimento mostraram que não houve alteração significativa do consumo desses alimentos entre homens e mulheres. Foi observado, entretanto, que o consumo de ácidos graxos saturados e colesterol das mulheres e dos homens eram superiores à recomendação ${ }^{26}$ antes e após a suplementação com o suco de laranja. Entre os indivíduos eutróficos e com sobrepeso, o consumo total de energia da dieta antes e depois do suco de laranja estava dentro da taxa de variação recomendada ${ }^{26}$ tanto em homens como em mulheres (Tabela 2).

A ingestão de vitamina $C$ teve aumento significativo para as mulheres eutróficas e com sobrepeso, embora o aumento para as últimas não tenha sido significante. Para os homens, a vitamina $C$ aumentou significativamente nos eutróficos e com sobrepeso, atingindo a recomendação ${ }^{26}$ nos indivíduos com sobrepeso após a suplementação do suco (Tabela 2). O ácido fólico foi aumentado significativamente com o suco somente nas mulheres eutróficas, embora nenhum grupo das mulheres alcançasse a recomendação de $400 \mu \mathrm{g} / \mathrm{d}^{26}$. Os homens, entretanto, mostraram uma ingestão adequada de ácido fólico antes e após o suco de laranja, embora apresentassem grande variação entre eles.

A análise dos lípides e das lipoproteínas sanguíneos mostrou que após o consumo de $750 \mathrm{~mL} /$ dia de suco de laranja houve uma redução significativa de $11,0 \%$ do colesterol total e de $15,0 \%$ do LDL-C nos homens. Para as mulheres, após o consumo do suco de laranja, foi verificada redução de 10,0\% para o colesterol total e 15,0\% para o LDL-C (Tabela 3). Porém, a concentração de apo B, após o consumo de suco de laranja, não mostrou modificação significativa (Tabela 3 ). Já que cada LDL apresenta apenas uma apo B-100, esse resultado sugere a manutenção no número de partículas de LDL no soro dos indivíduos analisados, embora com menor teor de colesterol no centro lipídico.

Tabela 2. Ingestão dietética de nutrientes, pré e pós o período de suplementação com suco de laranja em homens e mulheres eutróficos $\left(\mathrm{IMC} \leq 25 \mathrm{~kg} / \mathrm{m}^{2}\right)$ e com sobrepeso (IMC $\left.>25 \mathrm{~kg} / \mathrm{m}^{2}\right)$, e comparação com as cotas dietéticas recomendadas (DRI-2006). Araraquara (SP), 2006

\begin{tabular}{|c|c|c|c|c|c|c|c|c|c|}
\hline \multirow{3}{*}{$\begin{array}{l}\text { Indivíduos } \\
\text { Nutrientes }\end{array}$} & & \multicolumn{4}{|c|}{ Mulheres } & \multicolumn{4}{|c|}{ Homens } \\
\hline & & \multicolumn{2}{|c|}{ Eutróficos } & \multicolumn{2}{|c|}{ Sobrepeso } & \multicolumn{2}{|c|}{ Eutróficos } & \multicolumn{2}{|c|}{ Sobrepeso } \\
\hline & & Pré & Pós & Pré & Pós & Pré & Pós & Pré & Pós \\
\hline Energia & $\mathrm{kcal} / \mathrm{d}$ & $1484 \pm 306$ & $1687 \pm 345$ & $1744 \pm 750$ & $2403 \pm 1139$ & $2892 \pm 1513$ & $2043 \pm 775$ & $2499 \pm 533$ & $2549 \pm 1200$ \\
\hline DRI & & \multicolumn{2}{|c|}{$1784-2164$} & \multicolumn{2}{|c|}{$1738-2310$} & \multicolumn{2}{|c|}{$2585-3015$} & \multicolumn{2}{|c|}{$2591-3439$} \\
\hline Proteína & $g / d$ & $87 \pm 26$ & $76 \pm 26$ & $86 \pm 27$ & $93 \pm 48$ & $135 \pm 32$ & $100 \pm 49$ & $136 \pm 45$ & $125 \pm 74$ \\
\hline DRI & & \multicolumn{2}{|c|}{$49-173$} & \multicolumn{2}{|c|}{$51-177$} & \multicolumn{2}{|c|}{$70-245$} & \multicolumn{2}{|c|}{$75-263$} \\
\hline Glicídios & $g / d$ & $204 \pm 35$ & $244 \pm 52^{*}$ & $252 \pm 110$ & $330 \pm 127$ & $429 \pm 250$ & $333 \pm 125$ & $.289 \pm 30$ & $332 \pm 163$ \\
\hline DRI & & \multicolumn{2}{|c|}{$222-320$} & \multicolumn{2}{|c|}{$228-329$} & \multicolumn{2}{|c|}{$315-455$} & \multicolumn{2}{|c|}{$339-489$} \\
\hline Lipídeos & $\mathrm{g} / \mathrm{d}$ & $44 \pm 20$ & $55 \pm 22$ & $44 \pm 34$ & $85 \pm 55$ & $89 \pm 60$ & $72 \pm 41$ & $.93 \pm 43$ & $91 \pm 52$ \\
\hline DRI & & \multicolumn{2}{|c|}{$44-77$} & \multicolumn{2}{|c|}{$45-79$} & \multicolumn{2}{|c|}{ 62- 109} & \multicolumn{2}{|c|}{$67-117$} \\
\hline AGS & $g / d$ & $14 \pm 8$ & $18 \pm 6$ & $14 \pm 12$ & $30 \pm 20$ & $25 \pm 13$ & $23 \pm 17$ & $32 \pm 15$ & $33 \pm 22$ \\
\hline DRI & & \multicolumn{2}{|c|}{$4-15$} & \multicolumn{2}{|c|}{$4-16$} & \multicolumn{2}{|c|}{$7-22$} & \multicolumn{2}{|c|}{$10-23$} \\
\hline Colesterol & $\mathrm{mg} / \mathrm{d}$ & $189 \pm 100$ & $232 \pm 164$ & $175 \pm 129$ & $308 \pm 71$ & $578 \pm 563$ & $271 \pm 310$ & $304 \pm 146$ & $386 \pm 240$ \\
\hline DRI & & \multicolumn{2}{|c|}{200} & \multicolumn{2}{|c|}{200} & \multicolumn{2}{|c|}{200} & \multicolumn{2}{|c|}{200} \\
\hline Vitamina C & $\mathrm{mg} / \mathrm{d}$ & $98 \pm 77$ & $199 \pm 52^{*}$ & $151 \pm 57$ & $222 \pm 64$ & $106 \pm 103$ & $203 \pm 32^{*}$ & $67 \pm 67$ & $207 \pm 54^{*}$ \\
\hline DRI & & \multicolumn{2}{|c|}{75} & \multicolumn{2}{|c|}{75} & \multicolumn{2}{|c|}{90} & \multicolumn{2}{|c|}{90} \\
\hline Folato & $\mu \mathrm{g} / \mathrm{g}$ & $192 \pm 98$ & $307 \pm 140^{*}$ & $217 \pm 98$ & $352 \pm 163$ & $406 \pm 159$ & $500 \pm 148$ & $314 \pm 207$ & $521 \pm 176$ \\
\hline DRI & & & 00 & 4 & 00 & & & & 00 \\
\hline
\end{tabular}

* diferença estatisticamente significativa, $p<0,05$. (Teste $t$ pareado). 
Devido ao conteúdo substancial de açúcares naturais do suco de laranja, como a frutose, glicose e sacarose $(64 \mathrm{~g}$ em $750 \mathrm{~mL}$ de suco de laranja), haveria um risco potencial de elevar a glicemia e os triacilgliceróis séricos nos indivíduos que estivessem consumindo essa quantidade de suco diariamente. Entretanto, não foi detectado aumento significativo na glicemia ou triacilgliceróis nos indivíduos desse experimento (Tabela 3). Além disso, a mensuração dessas variáveis bioquímicas antes e após a suplementação não excederam os valores recomedados ${ }^{29}$.

Medidas do HDL-C e apo A-I após o consumo de suco de laranja mostraram uma redução significativa de $9,0 \%$ e $16,0 \%$ nos homens e $6,0 \%$ e $21,0 \%$ nas mulheres, respectivamente (Tabela 3). Porém, o tamanho médio das partículas de HDL não foi alterado com a suplementação com o suco de laranja, indicando não haver modificação do conteúdo central das partículas de HDL, representado principalmente pelo éster de colesterol.

A análise da atividade da enzima PON1 mostrou redução não significativa de 20,0\% após a suplementação com o suco de laranja tanto em homens como em mulheres. Embora essa redução tenha ocorrido em 80,0\% dos indivíduos da amostra, não houve diferença significativa antes e de- pois do período de suplementação por causa do amplo desvio-padrão verificado, comum na mensuração desse parâmetro ${ }^{28}$.

\section{I S C U S S Ã O}

A avaliação bioquímica dos lípides séricos após a suplementação com o suco de laranja mostrou que o consumo frequente desse alimento favorece a redução das concentrações de colesterol total e de LDL-C. Estudos prévios mostraram que o consumo de suco de laranja pelo homem ${ }^{4,10,11}$ e o de flavonóides cítricos por animais de experimentação $0^{8,12}$ reduziram o colesterol total e de LDL, apontando ainda benefícios adicionais, como redução dos níveis de proteína $C$ reativa, de radicais livre, da peroxidação lipídica e de outros marcadores do estresse oxidativo ${ }^{3}$, além da redução de alguns marcadores inflamatórios ${ }^{9}$. Além disso, os flavonóides cítricos promoveram redução da biossíntese de colesterol hepático, pela inibição da atividade da HMG CoA redutase e ACAT em ratos alimentados com dieta rica em colesterol $^{12}$. A redução na atividade da ACAT leva à menor esterificação do colesterol hepático disponível para a formação das Lipoproteínas de Densidade Muito Baixa (VLDL), resultando, assim, na redução da secreção de VLDL do fígado ${ }^{30}$.

Tabela 3. Dosagens bioquímicas no soro de glicose, lípides, apolipoproteínas, enzima paraoxonase 1 (PON1) e ta-manho da partícula de HDL em homens e mulheres no início (pré) e final (pós) do período de suplementação dietética com suco de laranja. Araraquara (SP), 2006.

\begin{tabular}{|c|c|c|c|c|c|c|c|c|}
\hline \multirow{4}{*}{$\begin{array}{l}\text { Variáveis bioquímica } \\
\text { do soro }(\mathrm{mg} / \mathrm{dL})\end{array}$} & \multicolumn{8}{|c|}{ Suplementação com suco de laranja } \\
\hline & \multicolumn{4}{|c|}{ Mulheres $(n=15)$} & \multicolumn{4}{|c|}{ Homens $(n=14)$} \\
\hline & \multicolumn{2}{|c|}{ Pré } & \multicolumn{2}{|c|}{ Pós } & \multicolumn{2}{|c|}{ Pré } & \multicolumn{2}{|c|}{ Pós } \\
\hline & M & DP & M & DP & M & DP & M & DP \\
\hline Glicose & 80,00 & 5,00 & 81,00 & 6,00 & 80,00 & 4,00 & 82,00 & 5,00 \\
\hline Triglicerídeos & 88,00 & 39,00 & 98,00 & 48,00 & 102,00 & 33,00 & 106,00 & 35,00 \\
\hline Colesterol total & 174,00 & 30,00 & 157,00 & $22,00^{*}$ & 182,00 & 41,00 & 162,00 & $37,00 *$ \\
\hline LDL-colesterol & 104,00 & 23,00 & 88,00 & $20,00^{*}$ & 117,00 & 39,00 & 99,00 & 35,00 * \\
\hline HDL-colesterol & 53,00 & 12,00 & 49,00 & $9,00^{*}$ & 45,00 & 9,00 & 42,00 & 8,00 * \\
\hline Apo A-I & 130,00 & 30,00 & 103,00 & $24,00^{*}$ & 112,00 & 15,00 & 94,00 & $16,00 *$ \\
\hline Apo B-100 & 85,00 & 25,00 & 91,00 & 17,00 & 91,00 & 31,00 & 92,00 & 32,00 \\
\hline PON 1, (nmol. $\left.\mathrm{min}^{-1} \cdot \mathrm{mL}^{-1}\right)$ & 56,00 & 44,00 & 45,00 & 30,00 & 52,00 & 31,00 & 42,00 & 23,00 \\
\hline Tamanho da HDL, (nm) & 9,09 & 0,52 & 8,79 & 0,86 & 8,68 & 0,63 & 8,93 & 0,61 \\
\hline
\end{tabular}

* valores com diferença estatisticamente significativa, $p<0,05$. Teste $t$ pareado. 
No presente estudo, a concentração dos triacilgliceróis e da glicose séricos não foram alterados pelo consumo de suco de laranja, mesmo com o consumo de $750 \mathrm{~mL} / \mathrm{dia}$, que acrescentou cerca de $64 \mathrm{~g} /$ dia de açúcares naturais ou $315 \mathrm{kcal} /$ dia por indivíduo. Esses resultados corroboram a pesquisa anterior com homens e mulheres normocolesterolêmicos ${ }^{10,11}$ e com dislipidemia ${ }^{31}$ que não mostraram alteração nos níveis de triacilgliceróis mesmo com a suplementação regular de um a três copos de suco de laranja. Os autores sugeriram que, embora seja reportada redução dos triacilgliceróis séricos em animais experimentais suplementados com flavonoides cítricos, no homem esta influência é controversa. A magnitude dos efeitos sobre os triacilgliceróis séricos parece depender primariamente de fatores como idade, sexo, nível basal de glicose, insulina e triacilgliceróis; presença de resistência à insulina e quantidade de frutose consumida na dieta ${ }^{32}$. Estudo recente reportou que o consumo de frutose ou de xarope de frutose durante duas semanas, num total de $20 \%$ a $25 \%$ da energia da dieta, não aumentou as concentrações de triglicerídeos em indivíduos saudáveis e com sobrepeso ou obesidade $^{33}$.

Em relação ao consumo dietético de lípides totais, gorduras saturadas e colesterol, que potencialmente afetam o colesterol plasmático e de LDL, foi verificado que os homens apresentavam consumo muito acima das recomendações ${ }^{26}$, enquanto as mulheres levemente acima. Não houve, entretanto, alteração do padrão de ingestão de gorduras durante o período experimental, o que sugere que as modificações observadas no perfil lipídico ocorreram independentemente da contribuição dos lípides da dieta. Porém, o consumo elevado de gorduras saturadas e colesterol, tanto em homens como em mulheres, reflete fortemente a ingestão de fontes animais na alimentação diária, com pequena presença de frutas e verduras. Portanto, o suco de laranja representou uma importante adição no valor nutricional das dietas, acrescentando vitamina C, flavanonas, potássio, ácido fólico, e outros importantes mi- cronutrientes também observados em estudos anteriores ${ }^{10-12,31,34}$.

Estudos conduzidos em animais e no homem têm mostrado que a suplementação dietética com vitamina $C$ reduz taxa de oxidação de LDL e diminui a formação da placa ateromatosa devido à capacidade antioxidante do ácido ascórbico no organismo ${ }^{12,34}$. A vitamina $C$ também foi associada com menores níveis de pressão arterial sistêmica em homens adultos que consumiam regularmente suco de laranja ${ }^{35}$. Outro estudo sugeriu ainda que a ação sinérgica da vitamina $C$ com os flavonóides presentes no suco cítrico promoveram o relaxamento vascular proporcionando a manutenção e o equilíbrio da pressão arterial $^{36}$.

Neste estudo foi verificada uma proporção elevada de indivíduos com sobrepeso e obesidade, sendo $36,0 \%$ de homens e $33,0 \%$ de mulheres, acompanhando a tendência à prevalência de adultos com excesso de peso na região sudeste do país. A distribuição da gordura corporal percentual foi idêntica à distribuição do IMC, sugerindo que todos os indivíduos com sobrepeso e obesos apresentavam excesso de massa gorda. A medida da circunferência da cintura confirmou ainda que esses mesmos participantes apresentavam maior risco de desenvolvimento de complicações relacionadas à obesidade, verificando-se média de $10 \mathrm{~cm}$ de circunferência abdominal acima dos valores seguros ${ }^{24}$ tanto para os homens quanto para as mulheres. É notório que medidas do IMC e da circunferência abdominal superiores aos valores limítrofes estabelecidos se associam positivamente com fatores de risco predisponentes para as doenças cardiovasculares ${ }^{37}$.

Como já mostrado na literatura, a aterogênese também está estreitamente correlacionada ao aumento nos níveis de peroxidação lipídica. Em condições de homeostase, a oxidação da LDL é provavelmente mínima devido tanto à presença de muitos antioxidantes dietéticos distribuídos no plasma e agregados a essa lipoproteína como também à remoção eficiente da LDL por meio do seu reconhecimento por receptores presentes abundantemente nas células hepáticas ${ }^{36}$. Con- 
tudo, essa situação pode ser modificada quando há aumento de espécies reativas de oxigênio ou nitrogênio, estabelecendo um quadro de estresse oxidativo. Uma vez que a modificação oxidativa da LDL nativa, decorrente desse balanço oxidativo, é um ponto chave na gênese da aterosclerose, a proteção dessa lipoproteína contra a oxidação pode ser uma estratégia eficaz para a prevenção e/ou para redução do risco de progressão dos eventos implicados na formação do ateroma. Sendo assim, a mensuração de marcadores de peroxidação lipídica figura como uma excelente ferramenta na caracterização de substâncias que agem como protetores tissulares e plasmáticos dos eventos oxidativos ${ }^{38}$.

A PON 1, um marcador bioquímico da peroxidação lipídica, teve sua atividade reduzida provavelmente devido ao aporte elevado de vitamina $C$ obtido pela ingestão de $750 \mathrm{~mL}$ de suco de laranja por dia. Além disso, experimentos sugerem que os flavonóides agem em sinergia com a vitamina $C$, potencializando seu efeito antioxidante nas lipoproteínas do sangue e prevenindo os danos da aterosclerose ${ }^{3}$, o que pode ter também contribuído para reduzir a ação da PON1. Os resultados de Rosenblat ${ }^{39}$ sugerem que a HDL aumenta a atividade da PON1 ao estabilizar a enzima. Neste trabalho houve redução significativa de partículas de HDL, o que pode ter influenciado na redução da atividade da PON1. Outra hipótese para a redução da atividade da PON1 está relacionada à redução de Apo A-l; já a maior parte da PON1 sérica está localizada na superfície da HDL e na Apo A-I. Assim, a deficiência dessa apolipoproteína em humanos reflete a redução da atividade sérica da PON 122.

\section{O N C L U S Ã O}

O consumo de suco de laranja não foi associado a variações no peso corporal, IMC, porcentagem de gordura e circunferência abdominal após a suplementação diária de $750 \mathrm{~mL}$ durante 60 dias. O suco de laranja reduziu o colesterol total e LDL-C, demonstrando propriedade hipocolesterolêmiande e antiaterogênica. A redução do HDL-C e da apo A-I foi associada à redução total do colesterol sanguíneo e à menor atividade oxidante no sangue devido ao elevado aporte de vitamina $\mathrm{C}$ e flavanonas cítricas.

O estudo do efeito da ingestão crônica e prolongada de suco de laranja, rico em hesperidina e vitamina $C$, demonstrou a ação hipolipemiante desse alimento natural, que levou à redução do risco de desenvolvimento de aterosclerose em homens e mulheres normolipidêmicos.

\section{OLABORADORES}

T.B. CÉSAR foi responsável pela elaboração do projeto de pesquisa, do protocolo experimental, obtenção de verba para a realização do experimento, supervisão do experimento e elaboração do texto final deste artigo. L.U. RODRIGUES foi responsável pela seleção dos voluntários, pela coleta de dados nutricionais, bioquímicos e antropométricos, análise e discussão dos resultados e participou ativamente na redação deste artigo. M.S.P. ARAÚJO colaborou como médica responsável do projeto de pesquisa junto ao Comitê de Ética da Unesp; auxiliou na elaboração dos critérios de seleção dos voluntários, na coleta dos dados bioquímicos e antropométricos e colaborou na revisão do presente artigo. N.P. APTEKMANN colaborou na confecção do protocolo do estudo, na análise estatística dos dados e discussão dos resultados.

\section{REFERÊ NCIAS}

1. Terao J. Dietary flavonoids as antioxidants. Forum Nutr. 2009; 61:87-94

2. Vinson JA, Liang $X$, Proch J, Hontz BA, Dancel J, Sandone N. Polyphenol antioxidants in citrus juices: in vitro and in vivo studies relevant to heart disease. Adv Exp Med Biol. 2002; 505:113-22.

3. Franke AA, Cooney RV, Henning SM, Custer LJ. Bioavailability and antioxidant effects of orange juice components in humans. J Agric Food Chem. 2005; 53(13):5170-78.

4. Rosa JM, Xian-Liu Z, Guthrie N. Effect of citrus flavonoids and tocotrienols on serum cholesterol levels in hypercholesterolemic subjects. Atern Ther. 2007; 13(6):44-8. 
5. Silalahi J. Anticancer and health protective properties of citrus fruit components. Asia Pacific J Clin Nutr. 2002; 11(1):79-84.

6. Benavente-Garcia O, Castillo J, Alcaraz M, Vicente $V$, Del Rio JA, Ortuño A. Beneficial action of citrus flavonoids on multiple cancer-related biological pathways. Curr Cancer Drug Targets. 2007; 7(8): 795-809.

7. Whitman SC, Kurowska EM, Manthey JA, Daugherty A. Nobiletin, a citrus flavonoid isolated from tangerines, selectively inhibits class $A$ scavenger receptor-mediated metabolism of acetylated LDL by mouse macrophages. Atherosclerosis. 2005; 178(1):25-32.

8. Kurowska EM, Manthey JA. Hypolipidemic effects and absorption of citrus polymethoxylated flavones in hamsters with diet-induced hypercholesterolemia. J Agric Food Chem. 2004; 52(10):2879-86.

9. Ghanim H, Mohanty P, Pathak R, Chaudhuri A, Sia $A L$, Dandona P. Orange juice or fructose intake does not induce oxidative and inflammatory response. Diabetes Care 2007; 30(6):1406-11.

10. Aptekmann NP, Cesar TB. Orange juice improved lipid profile and blood lactate of overweight middleaged women subjected to aerobic training. Maturitas. 2010 Aug 20. [Epub ahead of print]

11. Kurowska EM, Spence JD, Jordan J, Wetmore S, Freeman DJ, Piché LA, et al. HDL-cholesterol-raising effect of orange juice in subjects with hypercholesterolemia. Am J Clin Nutr. 2000; 72(5): 1095-100.

12. Bok SH, Lee SH, Park YB, Bae KH, Son KH, Jeong $\mathrm{TS}$, et al. Plasma and hepatic cholesterol and hepatic activities of 3-hydroxy-3-methyl-glutarylCoA reductase and Acyl CoA: cholesterol transferase are lower in rats fed citrus peel extract or a mixture of citrus bioflavonoids. J Nutr. 1999; 129(6):1182-5.

13. Knekt $P$, Kumpulainen J, Järvinen $R$, Rissanen $H$, Heliövaara M, Reunanen A, et al. Flavonoid intake and risk of chronic diseases. Am Clin Nutr 2002; 76(3):560-8.

14. Levine M, Rumsey SC, Daruwala R, Park JB, Wang $Y$. Criteria and recommendations for vitamin $C$ intake. J Am Med Assoc 1999; 281(15):1415-23.

15. Helmersson J, Arnlöv J, Larsson A, Basu S. Low dietary intake of beta-carotene, alpha-tocopherol and ascorbic acid is associated with increased inflammatory and oxidative stress status in a Swedish cohort. Br J Nutr. 2009; 101(12):1775-82.

16. Engler MM, Engler MB, Malloy MJ, Chiu EY, Schloetter MC, Paul SM, et al. Antioxidants vitamins $\mathrm{C}$ and $\mathrm{E}$ improve endothelial function in children with hyperlipidemia: Endothelial Assessment of Risk from Lipids in Youth (EARLY) Trial. Circulation. 2003; 108(9):1059-63.

17. Riso P, Visioli F, Gardana C, Grande S, Brusamolino A, Galvano F, et al. Effects of blood orange juice intake on antioxidant bioavailability on different markers related to oxidative stress. J Agric Food Chem. 2005; 53(4):941-7.

18. Sánchez-Moreno C, Cano MP, Ancos B, Plaza L, Olmedilla B, Granado F, et al. Effect of orange juice intake on vitamin $C$ concentrations and biomarkers of antioxidant status in humans. Am J Clin Nutr. 2003; 78(3):454-60.

19. Sánchez-Moreno C, Cano MP, Ancos B, Plaza L, Olmedilla B, Granado F, et al. High-pressurized orange juice consumption affects plasma vitamin $C$, antioxidative status and inflammatory markers in healthy humans. J Nutr. 2003; 133(7):2204-9.

20. Fuhrman B, Volkova N, Coleman R, Aviram M. Grape powder polyphenols attenuate atherosclerosis development in apolipoprotein E deficient (E0) mice and reduce macrophage atherogenicity. J Nutri. 2005; 135(4):722-8.

21. Getz GS, Reardon CA. Paraoxonase, a cardioprotective enzyme: continuing issues. Curr Opin Lipidol. 2004; 5(3):261-7.

22. James RW, Deakin SP. The importance of high-density lipoproteins for paraoxonase-1 secretion, stability, and activity. Free Rad Biol \& Med. 2004; 37(12):1986-94.

22. Rozenberg O, Rosenblat M, Coleman R, Shih DM, Aviram M. Paraoxonase (PON1) deficiency is associated with increased macrophage oxidative stress: studies in PON1-knockout mice. Free Rad Biol Med. 2003; 34(6):774-84.

24. Janssen I, Heymsfield SB, Allison DB, Kotler DP, Ross R. Body mass index and waist circumference independently contribute to the prediction of nonabdominal, abdominal subcutaneous and visceral fat. Am J Clin Nutr. 2002; 75(4):683-8.

25.Dâmaso AR. Métodos de avaliação da composição corporal. In: Nutrição e metabolismo na prevenção de doenças. Rio de Janeiro: Médica e Científica, 2001. p.125-52.

26. Institute of Medicine Food and Nutrition Board. Dietary reference intakes research synthesis. Workshop Summary. Washington: National Academy Press; 2006, p.310.

27. Friedewald WT, Levy RI, Fredrickson DS. Estimation of the concentration of low-density lipoprotein cholesterol in plasma, without use of the preparative ultracentrifuge. Clin Chem. 1972; 18(6): 499-502. 
28. Lima ES, Maranhão RC. Rapid, simple laser-light-scattering method for HDL particle sizing in whole plasma. Clin Chem. 2004; 50(6):1086-8.

29. Sociedade Brasileira de Cardiologia. IV Diretrizes Brasileiras sobre Dislipidemias e Prevenção da Aterosclerose do Departamento de Aterosclerose da Sociedade Brasileira de Cardiologia. Arq Bras Cardiol. 2007; 88(1):1-19.

30. Carr TP, Parks JS, Rudel LL. Hepatic ACAT activity in African green monkeys is highly correlated to plasma LDL cholesteryl ester enrichment and coronary artery atherosclerosis. Arteriosc Thrombosis. 1992; 12(11):1274-83.

31. Garcia ACDB, Bonifácio NP, Vendramine RC, César TB. Influência do suco de laranja nos lípides sanguíneos e na composição corporal de homens normais e com dislipidemia. Nutrire. 2008; 33(2): $1-11$.

32. Kurowska EM, Borradaile NM, Spence JD, Carroll KK. Hypocholesterolemic effects of dietary citrus juices in rabbits. Nutr Res. 2000; 20(1):121-9.

33. Stanhope KL, Griffen SC, Bair BR, Swarbrick MM, Keim NL, Havel PJ. Twenty-four-hour endocrine and metabolic profiles following consumption of highfructose corn syrup-, sucrose-, fructose-, and glucose-sweetened beverages with meals. Am J Clin Nutr. 2008; 87(5):1194-203.

34. Sprecher DL, Pearce GL. Fiber-multivitamin combination therapy: a beneficial influence on low-density lipoprotein and homocysteine. Metabolism. 2002; 51(9):1166-70.

35. Bonifácio NP, César TB. Influência da ingestão crônica do suco de laranja na pressão arterial e composição corporal. Rev Bras Hipert. 2009, 16(2): 76-81.

36. Libby P. Inflammation in atherosclerosis. Nature 2002; 420(6917):868-74.

37. Mooradian AD, Haas MJ, Wehmeier KR, Wong NC. Obesity-related changes in high-density lipoprotein metabolism. Obesity. 2008; 16(6):1152-60.

38. Cascieri MA. The potential for novel antiinflammatory therapies for coronary artery disease. Nature Reviews. Drug Discovery. 2002; 1(2):122-30.

39. Rosenblat M, Karry R, Aviram M. Paraoxonase 1 (PON1) is a more potent antioxidant and stimulant of macrophage cholesterol efflux, when present in $\mathrm{HDL}$ than in lipoprotein-deficient serum: relevance to diabetes. Atherosclerosis. 2006; 187(1):74-81.

Recebido em: 15/9/2008

Versão final reapresentada em: 8/6/2009

Aprovado em: 11/11/2009 
